\title{
Fluctuations and Correlations in Sandpile Models
}

\author{
Alain Barrat, ${ }^{1}$ Alessandro Vespignani, ${ }^{2}$ and Stefano Zapperi ${ }^{3}$ \\ ${ }^{1}$ Laboratoire de Physique Théorique, * Bâtiment 210, Université de Paris-Sud, 91405 Orsay Cedex, France \\ ${ }^{2}$ The Abdus Salam International Centre for Theoretical Physics (ICTP), P.O. Box 586, 34100 Trieste, Italy \\ ${ }^{3}$ PMMH-ESPCI, 10 rue Vauquelin, 75231 Paris Cedex 05, France
}

(Received 11 March 1999)

\begin{abstract}
We perform numerical simulations of the sandpile model for nonvanishing driving fields $h$ and dissipation rates $\epsilon$. Unlike simulations performed in the slow driving limit, the unique time scale present in our system allows us to measure unambiguously the response and correlation functions. We discuss the dynamic scaling of the model and show that fluctuation-dissipation relations are not obeyed in this system.

PACS numbers: 45.70.Ht, 05.70.Ln
\end{abstract}

The sandpile automaton [1] is one of the simplest models of avalanche transport, a phenomenon of growing experimental and theoretical interest. In the model, introduced by Bak, Tang, and Wiesenfeld (BTW) [1], grains of "energy" are injected into the system. Open boundary conditions [1] or bulk dissipation ensure a balance between input and output flow and allow for a nonequilibrium stationary state. In the limit of slow external driving and small dissipation, which corresponds to an infinite time scale separation between driving and response, the model displays a highly fluctuating avalanche behavior, indicative of a critical point. Despite the impressive theoretical effort devoted to understanding the critical behavior of the model [2-4], several important issues still remain to be addressed.

Numerical simulations are usually performed under slow driving and boundary dissipation, since the limit of infinite time scale separation is easily implemented in the computer and provides a simple way to access the avalanche critical behavior [5-9]. However, due to the presence of two infinitely separated time scales, an unambiguous definition of dynamic response and correlation functions is not possible [10]. This hinders a clear characterization of the nonequilibrium stationary state in terms of static and dynamic response and correlation functions. Evaluation of these quantities helps to elucidate the nature of the critical point and provides a test of fluctuation-dissipation relations, at least in some weaker sense. Recently, it has been proposed to interpret the behavior of sandpile models in analogy with other nonequilibrium critical phenomena, such as absorbing phase transitions [11], driven interfaces in random media [12-14], and branching processes [15]. These theoretical studies suggest new ways to perform numerical simulations in which a unique time scale is considered $[11,14,16]$.

In this Letter, we present numerical simulations of the sandpile model for different driving rates $h$ and study how the system approaches the critical point when $h \rightarrow 0$. In this way, we are able to measure quantities that are not accessible in the time scale separation regime. The local density of active sites, which can be identified as the order parameter of the model [16], is homogeneous only in the case of bulk dissipation. For boundary dissipation, it displays a marked curvature that was anticipated in Refs. $[11,14]$ and could explain several scaling anomalies found in the BTW model. The energy landscape is instead homogeneous in both cases and its statistical properties do not depend on the dissipation rate $\epsilon$ in the limit $h \rightarrow 0$.

We measure correlation and response functions in time and space domains and observe the scaling of the related characteristic lengths and times. We find two different characteristic times, implying that fluctuation-dissipation relations are not obeyed. We observe, however, a welldefined scaling behavior and the value of the critical exponents are in agreement with recent large scale numerical simulations of slowly driven sandpiles [7-9]. Finally, the present numerical analysis opens the road to future studies to resolve some long-standing problems such as the precise identification of universality classes for these models [8].

In sandpile models [1], each site $i$ of a $d$-dimensional lattice bears an integer variable $z_{i} \geq 0$, which we call energy. At each time step an energy grain is added on a randomly chosen site. When a site reaches or exceeds a threshold $z_{c}$ it topples: $z_{i} \rightarrow z_{i}-z_{c}$ and $z_{j} \rightarrow z_{j}+1$ at each of the $g$ nearest neighbors (nn) of $i$. Each toppling can trigger nn to topple and so on, generating an avalanche. The original BTW model is conservative and energy is dissipated only at the boundary, i.e., energy grains from toppling boundary sites flow out of the system. Infinitely slow driving is implicitly built into the model: during the avalanche the energy input stops, until the system is again quiescent (no active sites are present), so that we can identify two distinct time scales $T_{d}$ and $T_{a}$, for driving and activity, respectively. A single driving time step can, in principle, be followed by an infinite number of avalanche time steps and $T_{a} / T_{d} \rightarrow 0$. For this reason, there are two possible definitions for the correlation function, depending on the choice of the scale used to measure time (slow or fast) $[10,17]$. 
Here we simulate the BTW sandpile model for a nonvanishing driving field: each site has a probability $h$ per unit time to receive an energy grain also if active sites are present in the system. This defines a unique time step for both driving and activity updating. The parameter $h$ sets the driving rate, and in the limit $h \rightarrow 0^{+}$we recover the slow driving limit; i.e., during the evolution of an avalanche the system does not receive energy. We consider two possible mechanisms for energy dissipation, (i) usual boundary dissipation and (ii) bulk dissipation, simulated by introducing the probability $\alpha$ that a toppling site loses its energy without transferring it to the neighbors, which corresponds to an effective average dissipation $\epsilon=\alpha z_{c}$. In case (ii), we impose periodic boundary conditions. We use two-dimensional lattices with linear sizes ranging from $L=64$ to $L=300$, and parameters in the range $10^{-6}<h<10^{-3}$ and $10^{-3}<\epsilon<10^{-2}$.

The order parameter in sandpile models is the density $\left\langle\rho_{a}\right\rangle$ [18] of active sites, whose energy is equal to or larger than $z_{c}[1,11,12,14,16]$. The dependence of the order parameter on the control parameters $h$ and $\epsilon$ is readily obtained by means of conservation arguments [16]: since energy is conserved in the stationary state, the incoming energy flux $\left\langle J_{\text {in }}\right\rangle=h L^{d}$ must be equal to the dissipated energy $\left\langle J_{\text {out }}\right\rangle=\epsilon\left\langle\rho_{a}\right\rangle L^{d}$. By equating the two fluxes we obtain $\left\langle\rho_{a}\right\rangle=h / \epsilon$. In systems with boundary dissipation, the effective dissipation rate scales with the system size as $\epsilon \sim L^{-\mu}$, with $\mu=2$ [16], yielding $\left\langle\rho_{a}\right\rangle \sim h L^{2}$. The model is critical just in the double limit $\epsilon \rightarrow 0$ and $h / \epsilon \rightarrow 0$ [16].

We study the behavior of the density of active sites in the system and measure the stationary average density of local energies, i.e., the density $\rho_{i}$ of sites with $i$ energy grains. In Fig. 1 we report the behavior of the densities as a function of $h / \epsilon$. For small values of $h / \epsilon$ we find $\left\langle\rho_{i}\right\rangle=\rho_{i}^{0}+c_{i} h / \epsilon+\mathcal{O}\left((h / \epsilon)^{2}\right)$, where $\rho_{i}^{0}$ are the values extrapolated from the limit $h \rightarrow 0^{+}$and are given by $\rho_{0}^{0}=0.075(1), \rho_{1}^{0}=0.176(1), \rho_{2}^{0}=0.307(1)$, and $\rho_{3}^{0}=0.442(1)$. These values are in excellent agreement with the exact values obtained for the slowly driven sandpile (with boundary dissipation) [3] and are independent of the dissipation rate. For $i>3$ we obtain $\rho_{i}^{0}=0$ and for small $h$ we observe $\left\langle\rho_{a}\right\rangle \simeq\left\langle\rho_{4}\right\rangle$, while for larger $h$ higher energy levels become populated and $\left\langle\rho_{a}\right\rangle$ has nonnegligible contributions coming from $\left\langle\rho_{i}\right\rangle$ with $i>4$. Finally, we confirm that $\left\langle\rho_{a}\right\rangle=h / \epsilon$ for the whole range of parameters. In the case of boundary dissipation we recover $\left\langle\rho_{a}\right\rangle \sim h L^{2}$.

To elucidate the differences between bulk and boundary dissipation, we measure the local density of active sites $\left\langle\rho_{a}(r)\right\rangle$. In the case of bulk dissipation the density profile is flat $\left\langle\rho_{a}(r)\right\rangle=\left\langle\rho_{a}\right\rangle$, while in the case of boundary dissipation we obtain a surface that can be well approximated by a paraboloid (see Fig. 2). This is due to the highly inhomogeneous dissipation which imposes a zero density of active sites on the lattice boundary and corresponds to an elastic interface pinned at the boundaries as discussed in

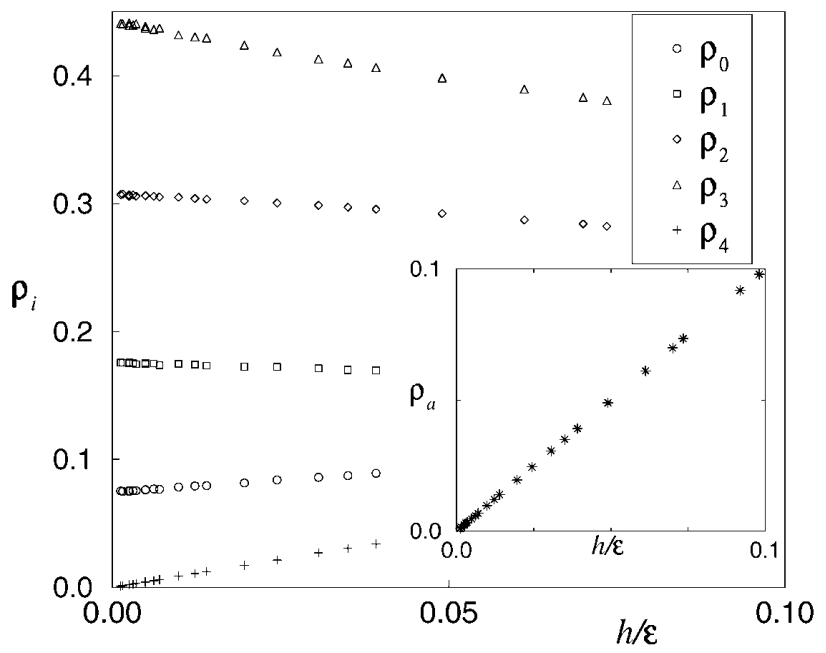

FIG. 1. Mean densities $\left\langle\rho_{i}\right\rangle$ of sites with $i$ energy grains vs $h / \epsilon$. Inset: mean density $\left\langle\rho_{a}\right\rangle$ of active sites vs $h / \epsilon$. Values of $h$ range from $10^{-6}$ to $10^{-3}$, and $\epsilon$ is between $10^{-3}$ and $10^{-2}$. We observe that $\left\langle\rho_{a}\right\rangle=h / \epsilon$ and that the various $\left\langle\rho_{i}\right\rangle$ depend on $h$ and $\epsilon$ only through the ratio $h / \epsilon$ (see text).

Refs. [11,14]. An inhomogeneous order parameter could give rise to anomalies in the avalanche exponents [7,9] or persistent deviations from simple scaling [19].

In order to obtain a quantitative description of the stationary state, we study the effect on the stationary density of a small perturbation in the driving field

$$
\Delta \rho_{a}(r, t)=\int \chi_{h, \epsilon}\left(r-r^{\prime}, t-t^{\prime}\right) \Delta h\left(r^{\prime}, t^{\prime}\right) d r^{\prime} d t^{\prime},
$$

where $\chi_{h, \epsilon}(r, t)$ is the local response function. In the limit $h \rightarrow 0^{+}$the integrated susceptibility $\chi \equiv \int d t d r \times$ $\chi_{h, \epsilon}(r, t)$ scales as the average avalanche size $\chi \sim\langle s\rangle$ and the time integrated response function scales as [16]

$$
\bar{\chi}_{h \rightarrow 0, \epsilon}(r) \equiv \int \chi_{h \rightarrow 0, \epsilon}(r, t) d t \propto \frac{1}{r^{d-2}} e^{-r / \xi},
$$

where $\xi$ is the characteristic length. Since $\chi=\partial \rho_{a} / \partial h$ and $\rho_{a}=h / \epsilon$, the response function diverges in the

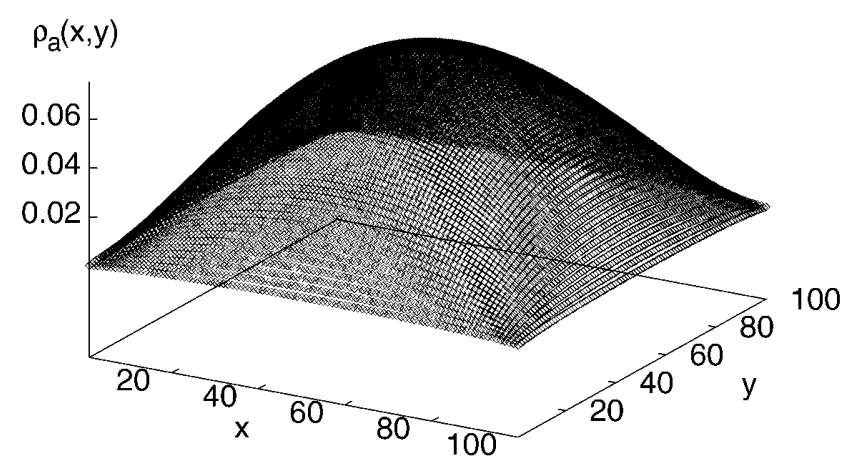

FIG. 2. Local density of active sites $\left\langle\rho_{a}(x, y)\right\rangle$, in the case of boundary dissipation; the linear lattice size is $L=100$, and $h=10^{-4}$. 
limit of vanishing driving and dissipation as $\chi=1 / \epsilon$. By noting that $\chi \sim \xi^{2}$, we obtain that $\xi \sim \epsilon^{-\nu}$ with $\nu=1 / 2$ [16]. These results hold in all dimensions due to conservation $[11,14,16]$.

To measure the response function, we drive the system in the stationary state with a given $h$ and we then add $n$ energy grains (i.e., $\Delta h=n / L^{2}$ ) on a given lattice site [20]. The time integrated response function is equivalent to the average difference of activity $\bar{\chi}_{h, \epsilon}(r)=$ $\Delta \rho_{a}(r)=\left\langle\rho_{a}(r)\right\rangle_{h+\Delta h}-\left\langle\rho_{a}(r)\right\rangle_{h}$, where $r$ denotes the distance from the perturbed site. We observe that this function decays exponentially as predicted by Eq. (2) and we measure the correlation length $\xi$ (see Fig. 3). In the case of bulk dissipation, for small driving fields the $\xi$ depends only on the dissipation rate and scales as $\xi \sim \epsilon^{-\nu}$, with $\nu=0.50 \pm 0.01$ (see Fig. 4). In the case of boundary dissipation, to evaluate $\bar{\chi}_{h, L}(r)$ we have to consider explicitly the spatial inhomogeneity of the stationary density: $\left\langle\rho_{a}(r)\right\rangle \neq h / \epsilon$. We also observe in this case that the integrated response function decays exponentially and defines a correlation length increasing linearly with the lattice size, i.e., $\xi \sim L$. This result does not agree with the anomalous scaling found in a continuous energy sandpile [17]. We perform analogous simulations in $d=3$ and find that Eq. (2) is still verified [21].

Furthermore, we study the response function in the time domain defined as $\tilde{\chi}_{h, \epsilon}(t) \equiv \int d r \chi_{h, \epsilon}(r, t)$ after a small variation $\Delta h$ of the driving field. Also, in this case, we obtain a clear exponential behavior defining the characteristic time scale $\tau$. For small driving field, $\tau$ scales as a function of the dissipation rate as $\tau \sim \epsilon^{-\Delta}$, with $\Delta=0.75 \pm 0.05$ (see Fig. 4). We then evaluate the dynamical exponent $z=\Delta / \nu=1.5 \pm 0.1$ relating time and spatial characteristic length: $\tau \sim \xi^{z}$. In the limit $h \rightarrow 0^{+}$, we expect that the critical exponents $\nu$ and $z$ express the divergence of avalanche characteristic size

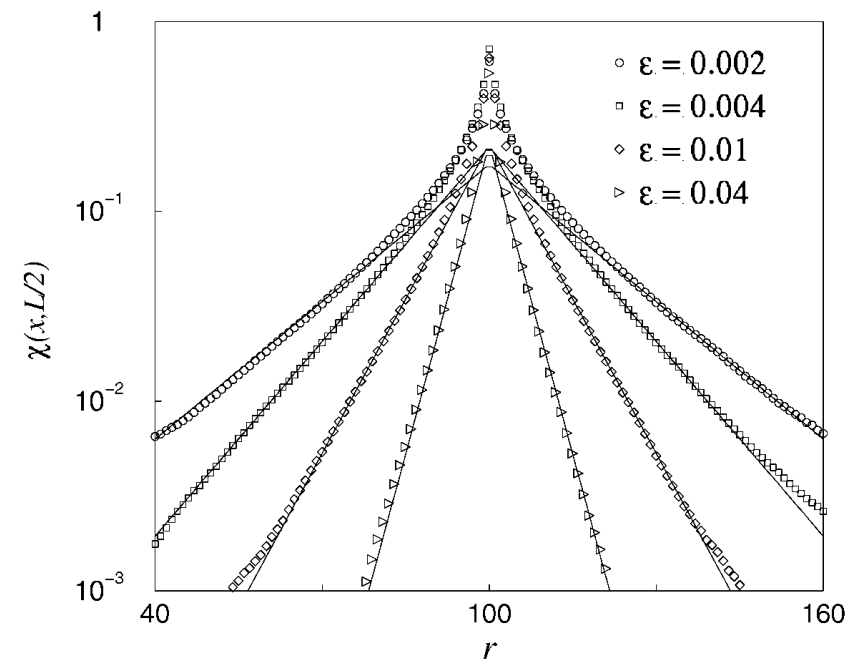

FIG. 3. Time integrated response function $\bar{\chi}_{h \rightarrow 0, \epsilon}$ to a constant perturbation as a function of $r$; the linear lattice size is $L=200$. The lines are exponential fits. and time, respectively. The numerical results confirm this observation [7-9]. For increasing values of $h$, the driving field enters the scaling form, which, however, goes to a constant for $h \rightarrow 0$ [21].

We now turn to the analysis of the correlation function defined as $C(r, t)=\left\langle\rho_{a}(r, t) \rho_{a}(0,0)\right\rangle-\left\langle\rho_{a}\right\rangle^{2}$. In previous simulations, performed in the slow driving limit, correlation functions were usually measured with respect to the slow time scale $[1,17,22]$, and the fast time scale was explored studying the avalanche propagation. The introduction of nonvanishing driving and dissipation allows us to bridge the gap between the two regimes. We study the correlation function in time and space domains and find an exponential decay at long times and distances [23], defining the correlation lengths $\xi_{c}$ and $\tau_{c}$ for space and time, respectively. The scaling of these correlation lengths is in agreement with the one obtained analyzing the response functions (i.e., $\xi_{c} \sim \epsilon^{-\nu}$, with $\nu \simeq 0.5$, and $\tau_{c} \sim \epsilon^{-\Delta}$, with $\Delta \simeq 0.75$ ) and confirms the existence of a unique critical behavior in time and space (see Fig. 4).

In order to clarify the interplay between slow and fast dynamical modes, we analyze fluctuation-dissipation relations. In equilibrium phenomena, the fluctuationdissipation theorem ensures that the response of the system to a small perturbation is related to the correlation function. In particular, the response function is given by

$$
\chi(t)=-\frac{1}{T} \frac{d C(t)}{d t}
$$

where $T$ is the temperature. Equation (3) is strictly verified only in equilibrium systems, but it has been recently generalized to some classes of nonequilibrium systems, namely systems displaying "aging" [24]. In those examples the fluctuation-dissipation relation provides an

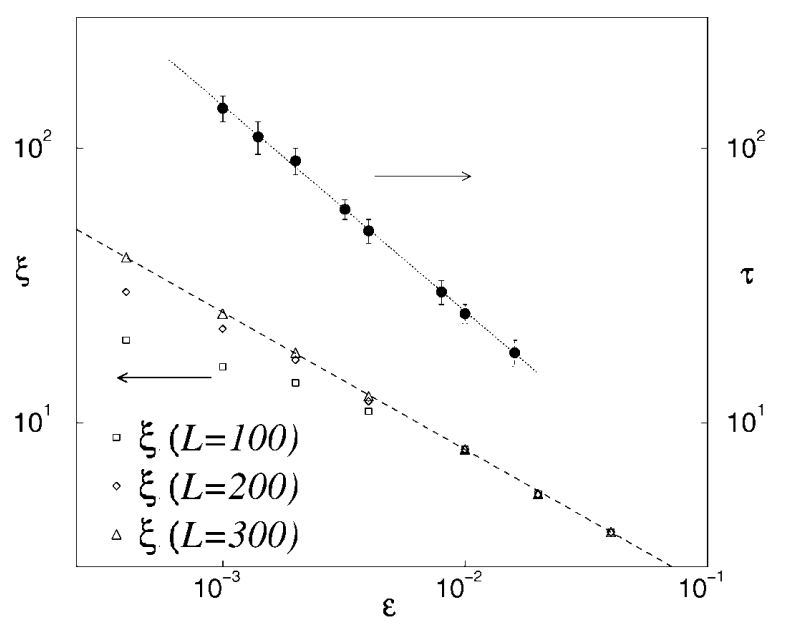

FIG. 4. Characteristic length $\xi$ and characteristic time $\lim _{h \rightarrow 0^{+}} \tau(h, \epsilon)$ estimated from the spatial response and correlation functions with bulk dissipation and for various system sizes and dissipation rates. For small dissipation rates $\xi \rightarrow \infty$, and larger lattice sizes must be used. The straight lines represent the best fits with slope $\nu=0.5$ and $\Delta=0.75$ for $\xi$ and $\tau$, respectively. 
information on an effective nonequilibrium temperature that rules the dynamical evolution of the system.

We test Eq. (3) and we find that the usual linear behavior does not hold. On the contrary, we show that the parametric plot of $\chi(t)$ versus $C(t)$ defines a power law behavior, as shown in the double logarithmic plot of Fig. 5. This is striking evidence that the fluctuation-dissipation relation does not hold in these systems. Since we are in the presence of two exponential functions, the linear behavior on the logarithmic scale is the signature of two different values for characteristic times $\tau_{c}$ and $\tau$ for the correlation and response functions, respectively. The slope indicates that the ratio between the two time scales is given by $\tau_{c} / \tau \simeq 0.4$ and does not depend on driving and dissipation rates. This observation reflects the fact that the correlation and the response times scale with the same exponents with respect to dissipation and define unambiguously the critical behavior of the model. In particular, it implies that the dynamical exponent $z \simeq 1.5$ is unique and can be estimated either by measuring avalanche distributions or the correlation functions. Previous simulations $[7,17]$ revealed two different dynamical exponents in the fast and slow time scales. These differences are probably due to the ambiguous definition of time in the infinite time scale separation limit.

Finally, it is interesting to compare our results with a recent work [25] showing that the stationary state of nonequilibrium threshold models, similar to the one studied here, can be described by Boltzmann statistics in the mean-field limit. The validity of the claim of Ref. [25] has been debated in the literature [26]. We measure fluctuationdissipation relations in a random neighbor sandpile model, which is described by mean-field theory, and find that fluctuation-dissipation relations are not satisfied [21], in disagreement with the conclusions of Ref. [25].

We thank A. Chessa, D. Dhar, R. Dickman, S. Franz, K. B. Lauritsen, E. Marinari, M. A. Muñoz, R. PastorSatorras, and A. Stella for comments and discussions.

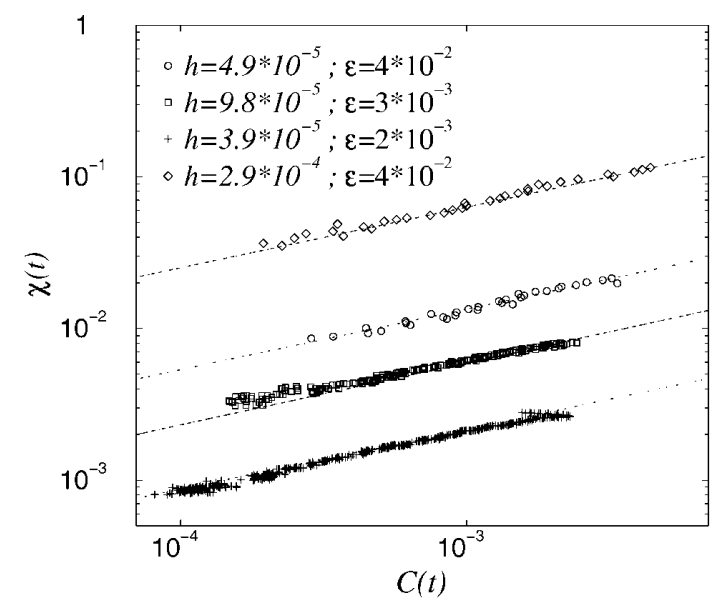

FIG. 5. Double logarithmic plot of $\chi(t)$ vs $C(t)$ for various values of $h$ and $\epsilon$; the slope of the straight lines is $\tau_{c} / \tau \simeq 0.4$.
A.V. and S.Z. acknowledge partial support from the European Network Contract No. ERBFMRXCT980183.

*Unité Mixte de Recherche UMR 8627.

[1] P. Bak, C. Tang, and K. Wiesenfeld, Phys. Rev. Lett. 59, 381 (1987); Phys. Rev. A 38, 364 (1988).

[2] D. Dhar, Phys. Rev. Lett. 64, 1613 (1990).

[3] V. B. Priezzhev, J. Stat. Phys. 74, 955 (1994).

[4] A. Díaz-Guilera, Phys. Rev. A 45, 8551 (1992); L. Pietronero, A. Vespignani, and S. Zapperi, Phys. Rev. Lett. 72, 1690 (1994).

[5] P. Grassberger and S. S. Manna, J. Phys. (Paris) 51, 1077 (1990); S. S. Manna, J. Stat. Phys. 59, 509 (1990); Physica (Amsterdam) 179A, 249 (1991).

[6] S. S. Manna, J. Phys. A 24, L363 (1991).

[7] S. Lübeck and K.D. Usadel, Phys. Rev. E 55, 4095 (1997); 56, 5138 (1997); S. Lübeck, ibid. 56, 1590 (1997).

[8] A. Chessa, H. E. Stanley, A. Vespignani, and S. Zapperi, Phys. Rev. E 59, R12 (1999).

[9] A. Chessa, E. Marinari, A. Vespignani, and S. Zapperi, Phys. Rev. E 57, R6241 (1998).

[10] T. Hwa and M. Kardar [Phys. Rev. A 45, 7002 (1992)] and A. Montakhab and J. M. Carlson [Phys. Rev. E 58, 5608 (1998)] discuss the role of driving in sandpile models and report numerical results for several quantities in the "fast" driven regime.

[11] A. Vespignani, R. Dickman, M. A. Muñoz, and S. Zapperi, Phys. Rev. Lett. 81, 5676 (1998); R. Dickman, A. Vespignani, and S. Zapperi, Phys. Rev. E 57, 5095 (1998).

[12] O. Narayan and A. A. Middleton, Phys. Rev. B 49, 244 (1994).

[13] M. Paczuski and S. Boettcher, Phys. Rev. Lett. 77, 111 (1996).

[14] K. B. Lauritsen and M. Alava (to be published).

[15] S. Zapperi, K. B. Lauritsen, and H. E. Stanley, Phys. Rev. Lett. 75, 4071 (1995).

[16] A. Vespignani and S. Zapperi, Phys. Rev. Lett. 78, 4793 (1997); Phys. Rev. E 57, 6345 (1998).

[17] A. Giacometti and A. Díaz-Guilera, Phys. Rev. E 58, 247 (1998).

[18] The bracket defines an average over different realizations.

[19] M. De Menech, A. L. Stella, and C. Tebaldi, Phys. Rev. E 58, R2677 (1998).

[20] Different ways of perturbing the system yield the same response function behavior and characteristic length [21].

[21] A. Barrat, A. Vespignani, and S. Zapperi (to be published).

[22] B. Kutnjak-Urbanc, S. Havlin, and H. E. Stanley, Phys. Rev. E 54, 6109 (1996).

[23] The correlation function sometimes shows periodic oscillations that can be described in mean-field theory [16], and will be reported in [21].

[24] See, e.g., L.F. Cugliandolo, J. Kurchan, and L. Peliti, Phys. Rev. E 55, 3898 (1997).

[25] J. B. Rundle, W. Klein, S. Gross, and D. L. Turcotte, Phys. Rev. Lett. 75, 1658 (1995).

[26] H.-J. Xu and D. Sornette, Phys. Rev. Lett. 78, 3797 (1997); J. B. Rundle, W. Klein, S. Gross, and D. L. Turcotte, ibid. 78, 3798 (1997). 